\title{
The effects of an exercise program on health-related quality of life in postpartum mothers: A randomized controlled trial
}

\author{
Megumi Haruna $^{1 *}$, Etsuko Watanabe ${ }^{1}$, Masayo Matsuzaki ${ }^{1}$, Erika Ota $^{1}$, Mie Shiraishi ${ }^{1}$, \\ Ryoko Murayama ${ }^{1}$, Mikako Yoshida ${ }^{1}$, SeonAe Yeo $^{2}$ \\ ${ }^{1}$ Department of Midwifery and Women's Health, Division of Health Sciences and Nursing, Graduate School of Medicine, The Uni- \\ versity of Tokyo, Tokyo, Japan; 'Corresponding Author: mharuna-tky@umin.ac.jp \\ ${ }^{2}$ School of Nursing, University of North Carolina at Chapel Hill, North Carolina, USA
}

Received 18 January 2013; revised 25 February 2013; accepted 2 March 2013

\section{ABSTRACT}

Background and Objective: There is controversy on the psychological effects of postpartum exercise. The study aimed to evaluate the effectiveness of a postpartum exercise program on healthrelated quality of life and psychological wellbeing. Methods: We conducted a randomized controlled trial in Tokyo, Japan. The intervention groups participated in ball-exercise classes (weekly exercise of $\mathbf{9 0}$ minutes for four weeks) at three months postpartum. The exercise class included the following: 1) greeting and warm-up; 2) aerobic exercise involving bouncing on an exercise ball 55 or $65 \mathrm{~cm}$ in diameter; 3) rest and selfintroduction; 4) stretching and cooling down. The primary outcome measure was health-related Quality of Life (QOL) assessed using the MOS Short-Form 36-Item Health Survey (SF-36v2). The secondary outcome measures were Rosenberg Self-Esteem Scale (RSES) and Edinburgh Postnatal Depression Scale (EPDS) scores. The intervention group was compared to the control group at four months postpartum. Results: of the 120 women screened, 110 women met the study criteria. Nine could not be included and the remaining 101 were allocated randomly into intervention and control groups (50 and 51 participants respectively). Analysis of covariance adjusting for baseline values indicated that the SF36 subscales of physical functioning $(p=$ $0.018)$ and vitality $(p=0.016)$ significantly improved in the intervention group compared to the control group, although there were no significant differences between the groups in the SF36 Physical Component Summary (PCS) and Mental Component Summary (MCS) scores. The RSES increased in the intervention group $(p=$
0.020) compared to the control group. No significant group differences were observed in EPDS scores. Conclusions: The postpartum exercise class program provided to healthy postpartum women appears to have contributed to promoting health-related QOL and self-esteem.

Keywords: Exercise; Health Related Quality of Life; Postpartum Depression; Randomized Controlled Trial; Self-Esteem

\section{INTRODUCTION}

A small number of women experience a major depressive episode during the first three months postpartum; most of these episodes have onset following delivery. A recent review found $7.1 \%$ of women experience major depression; including minor depressive episodes, the 3month period prevalence rate in this review was $19.2 \%$ [1]. In a Japanese study, the prevalence of major depression was $4.3 \%$ and the prevalence of mental disorder including minor depressive episode was $10.6 \%$ within three months postpartum [2]. Furthermore, many of those who are not diagnosed with depression complain of mild mood disorder or feelings of anxiety. Even postpartum mothers who experienced normal pregnancy and delivery, have a risk of worsening psychological wellbeing, although prevalence of postpartum Post-Traumatic Stress Disorder (PTSD) is higher among women after pregnancy complications such as preeclampsia [3].

A previous study suggested that mothers' feelings of loneliness related to positive scores (nine points and above) on the Edinburgh Postnatal Depression Scale (EPDS) at three months postpartum [4]. Therefore, it is important to prevent social isolation and mental disorder, and improve mental health among postpartum mothers. In Japan, $69.2 \%$ of child-rearing families have a nuclear 
family structure and $72.5 \%$ of mothers of infants do not work [5], so many mothers spend most of their time at home after childbirth to care for their babies. Therefore, they tend to be socially isolated and are susceptible to mental health problems. There is also a need to provide opportunities for mothers to be together.

Meanwhile, physical exercise is believed to improve mild depression, anxiety, and feelings of discomfort. Previous studies have shown that physical exercise intervention could help improve the mood and well-being of patients with depressive disorder [6]. It is therefore expected that the provision of an exercise program as part of postpartum care can help reduce mental health problems. Most studies related to the benefits of postpartum exercise on mental health have targeted womedn with higher EPDS scores and have shown the effect of exercise in reducing depressive symptoms in this population [7-10].

A few recent studies have focused on the psychological well-being of healthy postpartum women. Norman et al. conducted an eight-week mother and baby program, including specialized exercise combined with parenting education $(n=62)$, compared with a control group $(n=$ 73). They found that mothers in the intervention group improved their level of well-being (Positive Affect Balance Scale) and reduced their depressive symptoms (EPDS score) more than those in the control group [11]. Cramp et al. examined the effect of a moderate aerobic exercise session (two 45-min exercise sessions) on feeling states when women $(n=23)$ exercised with their baby present compared to without their baby present. The findings suggested that there were no differences in the Exercise-induced Feeling Inventory when women exercised with or without their babies. However, participants reported pre- to post-exercise improvements in feeling states and the State Anxiety Scale of the StateTrait Anxiety Inventory (STAI) [12].

There are a few studies showing that exercise substantially improves Quality of Life (QOL) in healthy populations such as pregnant women, postmenopausal women and elderly women. Montoya Arizabaleta et al. demonstrated that pregnant women $(n=64)$ in a 3-month supervised exercise program of primary aerobic exercise had improved their health-related QOL more than the control group [13]. Martin et al. showed that higher dose of exercise was associated with larger improvements in mental and physical aspects of QOL among 430 sedentary postmenopausal women [14]. Devereux et al. showed that a water-based exercise and self-management program produced significant beneficial changes in QOL among women 65 years and over $(n=50)$ [15]. However, little is known about the impact of exercise programs on health-related QOL during the postpartum period.

In this study, we designed a feasible aerobic exercise program which is expected to prevent isolation for postpartum mothers at three months after childbirth, and assessed its effect on health-related QOL and self-esteem using a randomized controlled trial (RCT).

\section{METHODS}

\subsection{Participants}

Recruitment started in December 2006 and was completed in August 2007. Participants were recruited from three sources; the University of Tokyo Hospital and two public health centers located in the same district. Women living in the Tokyo area in Japan, signed up for this study until one month postpartum. Women voluntarily applied for participation in the exercise class. Eligible women were more than 20 years of age without any medical complications who expected to give birth between January and July 2007. Women with restricted physical activity or suffering from psychiatric disorders were excluded from the study.

\subsection{Study Protocol}

We used a randomized controlled trial design to test the psychological outcome of exercise class in postpartum women. Two researchers not involved in the allocation process carried out the permutated block randomization (MM and EO). The ratio of randomization between the exercise and control group was 1:1 allocation using random block size of 4 .

Blinding of participants and the exercise instructors was not possible as participants were allocated to the exercise group or control group. All outcomes were assessed without blinding to the intervention. Data entry personnel were blinded to random assignment and the data was analyzed by a non-blinded assessor.

Of the 120 women at study entry, 110 women were eligible and agreed to participate in the study. They were allocated into the intervention group or control group using random numbers. However, nine women refused to participate at two months postpartum, and thus a total of 101 women were enrolled in the study (intervention group; $\mathrm{n}=50$, control group; $\mathrm{n}=51$ ).

At two months postpartum, researchers checked the health history, general health status, blood pressure and pulse rate to confirm eligibility for participation in the study. Age, height, pre-pregnancy weight, medical history, reproductive history and family history were recorded from maternal and child health records. The women completed a self-administered questionnaire that included demographic information such as schooling history, work status, regular exercise, smoking and drinking habits. The outcome measures were assessed at two months postpartum (baseline) and at four months postpartum (outcome). Of the 101 women who completed the 
baseline questionnaire, 95 finished the study. Of the 50 participants of the intervention group, four women did not attend the exercise classes, but of these three women still completed the outcome questionnaire. Figure $\mathbf{1}$ is a flow diagram of participants in the trial. The participants in the control group were offered the program after the outcome measurement.

The protocol was approved by the institutional review board of the University of Tokyo. All women provided written informed consent. The study was conducted in accordance with the principles of the Declaration of Helsinki.

\subsection{Postpartum Exercise Intervention}

Women in the intervention group participated in an exercise program that provided exercise classes four times weekly for four weeks, 90 minutes each, at three months postpartum. In each class, approximately 10 women participated and two expert exercise instructors familiar with postpartum exercise ensured the validity of the exercise program. The exercise class included the following: 1) greeting and warm-up, (15 minutes); 2) aerobic exercise (50 - 60 minutes) where the participant sits and bounces on an exercise ball (Gymnic, Italy) measuring $55 \mathrm{~cm}$ or $65 \mathrm{~cm}$ in diameter; 3) rest, self-introduction or partner-up communication (5 minutes); and 4) muscular stretching and cool down (15 - 20 minutes).

The participants were able to attend the class either with or without their babies. If they attended the class with their babies, babies were laid down in the center of the circle formed by the participants, and the mothers were able to hold their babies, change their diaper or breast-fed the babies at any time. The exercise instructors regulated in a flexible manner the time allocated to the aerobic exercise and the stretching and cool down component varied in length according to abilities of the participants. During the exercise, each participant had a chance to communicate with other participants.

\subsection{Main Outcome Measures}

The following three scales were evaluated.

- MOS Short-Form 36-Item Health Survey version 2 (SF-36v2): The SF-36 is a multi-purpose, comprehensive health survey with only 36 items for healthrelated QOL measures. The original version was developed in the United States, and it has been translated in more than 50 countries, and the reliability and validity of the Japanese version have been studied [16]. We used the standard (four-week) recall versions. Total score ranges from a minimum of 0 to a maximum of 100 , with higher scores indicating better health status. Three subscales [physical functioning, role-physical, and bodily pain] correlate most highly with the physical component and contribute most to the scoring of the Physical Component Summary (PCS) measure [17]. The mental component correlates most highly with the [mental health, roleemotional, and social functioning] scales, which also contribute most to the scoring of the Mental Component Summary (MCS) measure. Three of the subscales [vitality, general health, and social functioning] correlate with both components.

- Rosenberg Self-Esteem Scale (RSES): This scale measured the state of self-esteem, which was developed by Rosenberg in 1965 [18]. A Japanese version of the self-esteem scale has been developed [19]: it consists of 10 items, with a total score ranging from a low of 10 to a high of 50 . Higher scores indicate a "higher state of self-esteem".

- EPDS Japanese version: the Japanese version has been examined and validated externally. The EPDS is a 10 -item scale and an internationally used screening scale for postpartum depression [20]. The scale has been translated and validated for use in Japan. The validation study on the Japanese version of the EPDS demonstrated a sensitivity of 0.75 and a specificity of 0.93 at an optimum cut-off score of 9 and above [21], which was used in the present study.

\subsection{Sample Size Estimation}

The total sample size of 106 women was estimated for an expected effect size of 0.55 and power of 0.8 at an alpha of 0.05 [22]. This would be sufficient to detect a five point difference between groups in the PCS score of SF-36v2 on completion of the intervention, assuming a SD of 9.1 [16]. Taking into consideration possible dropouts, we recruited 120 women for the study.

\subsection{Statistical Analysis}

Descriptive statistics including means and standard deviations for quantitative variables and proportions for categorical variables were calculated for all baseline variables. The equivalence of control and intervention group was tested at baseline using unpaired t-test, chisquare test or Fisher's exact test. For each participant who completed the follow-up assessments, the changes in each outcome were calculated (i.e. post-score minus pre-score). We used one-way analysis of covariance (ANCOVA) to compare the groups with outcome data at four months postpartum in both the eight subscales and summary scores of SF-36v2, RSES and EPDS controlling for baseline values. The analyses were conducted on an intention-to-treat (ITT) basis for the outcome variables of the entire sample. We also conducted secondary analysis of 44 women who actually attended 


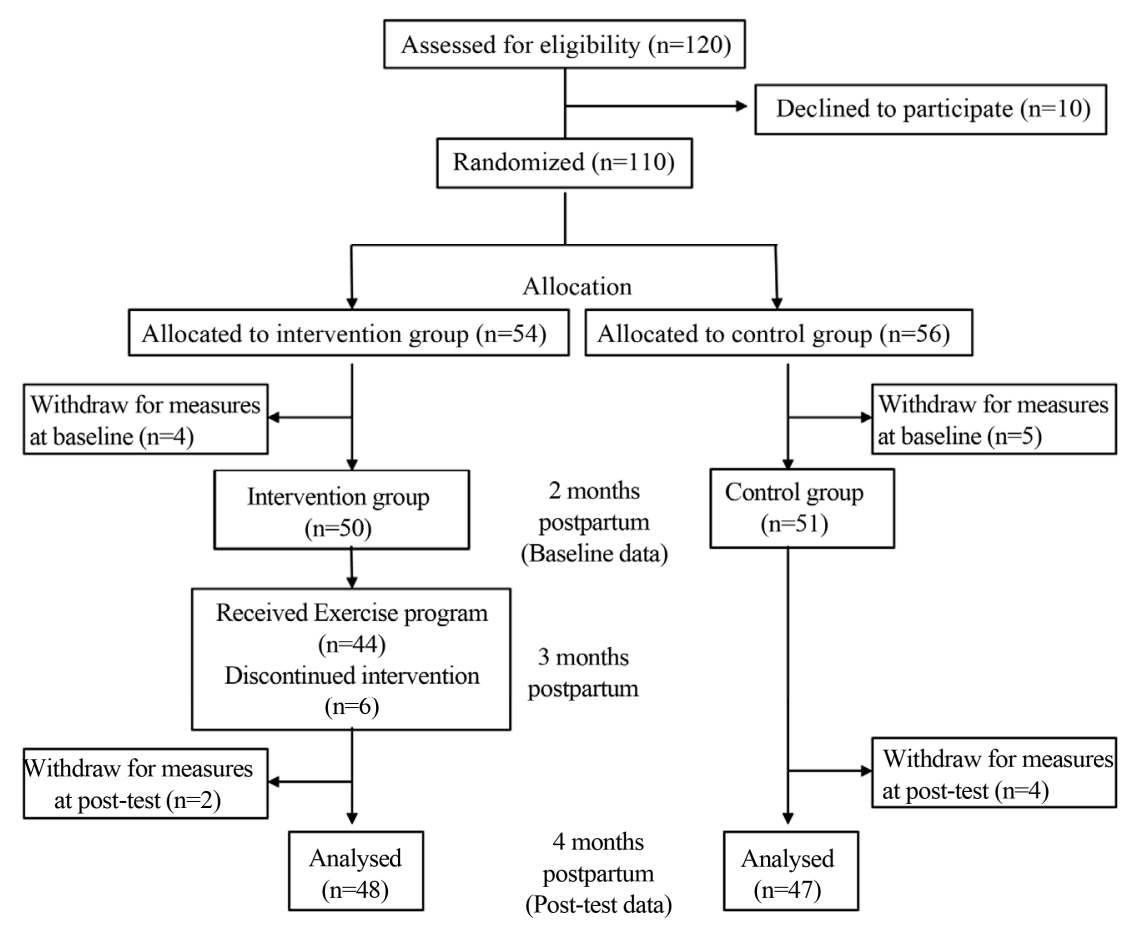

Figure 1. Flow diagram of participants through the study.

the classes twice or more and 47 women who completed the post-test evaluation in the control group. All $p$ values were 2-sided and $\mathrm{p}<0.05$ was considered statistically significant. The SPSS version 16.0 statistical software package (SPSS Japan Inc.) was used for statistical analyses.

\section{RESULTS}

\subsection{Demographic Data}

There were no significant differences between the two groups in terms of age and other demographic parameters. The two groups were well balanced with respect to socio-demographic characteristics (Table 1). Their babies were also in good health.

\subsection{Class Attendance Rate}

Finally, 48 women were followed through four months postpartum among 50 participants who were allocated to intervention group. Subsequently, the data for 48 women were analyzed. However, actual attendance at the class was 44 women and the attendance rate was $88.0 \%$.

\subsection{Comparison of SF-36v2 Scores of the Two Groups}

No baseline differences in SF-36v2 scores were apparent between intervention and control groups. At four months after childbirth, there was no significant difference in the PCS of SF-36v2 score between the two groups. ANCOVA analyses identified the exercise group improved significantly more than the control group in two of the eight subscales within the SF-36v2 score: the Physical Functioning $(\mathrm{PF})$ domain $(\mathrm{p}=0.018)$ and the Vitality (VT) domain $(\mathrm{p}=0.016)$. The MCS summary score and other subscales showed no significant difference due to the exercise intervention (Table 2).

\subsection{Comparison of EPDS Scores of the Two Groups}

At baseline, no significant differences were seen in the rate of women scoring $\geq 9$ on the EPDS score. The mean of EPDS score was higher in the control group than in the intervention group at baseline $(p=0.028)$. The mean of EPDS scores were reduced in both of intervention group and control group, but there were no differences between the two groups at outcome measurements (Table 2). Although we re-analyzed our data excluding women with high EPDS scores of 13.0 and above at baseline, there was no change in the results of outcome measurements.

\subsection{Secondary Analysis}

We conducted additional analysis of 44 women who actually attended the classes twice or more and 47 women who completed the post-test evaluation in the control group. ANCOVA analyses identified that the 44 women in the exercise intervention group showed significantly greater improvements than the 47 women in 
Table 1. Characteristics of participants of the intervention and control groups at baseline.

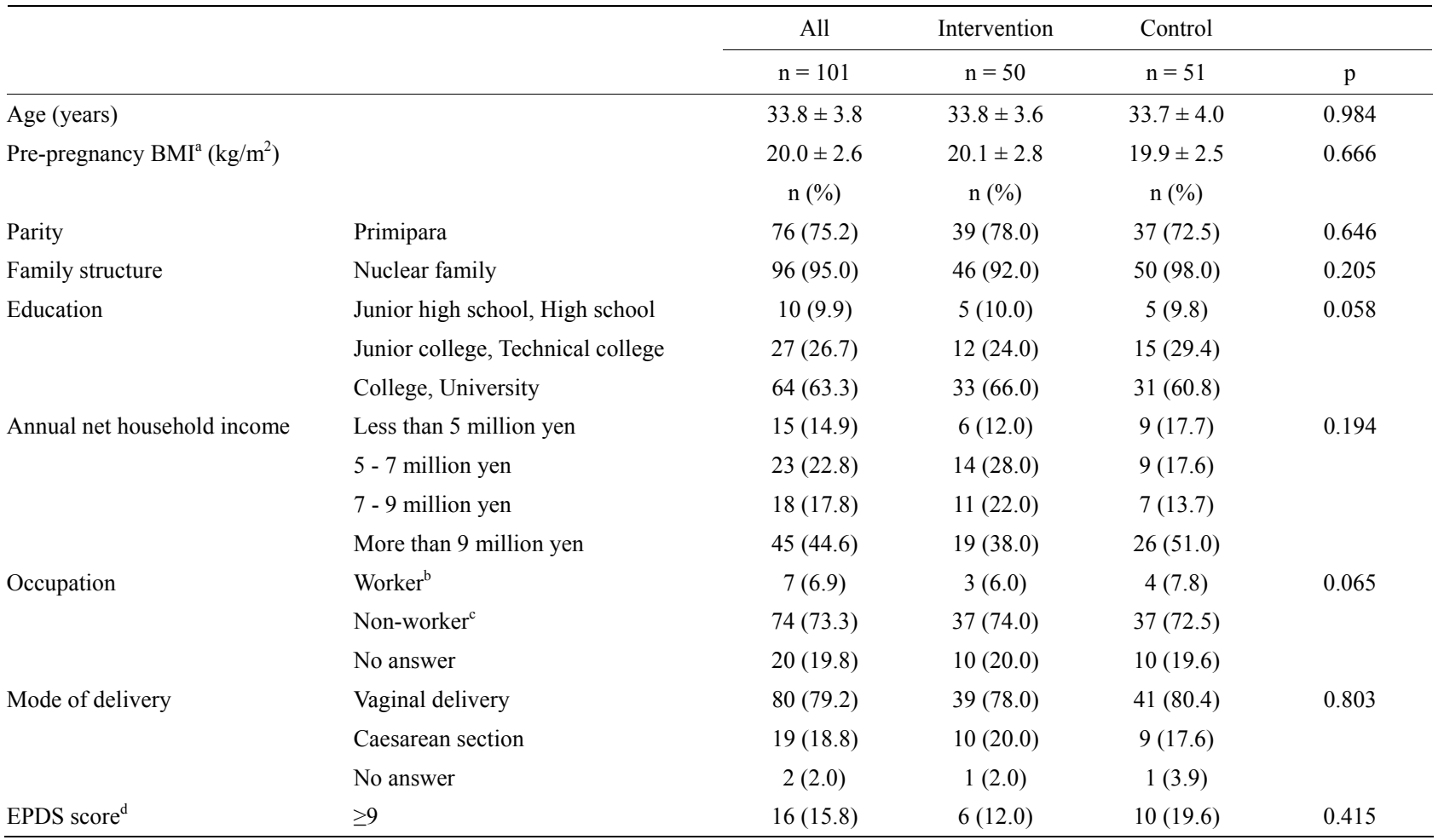

Data are mean \pm SD or n (\%), ${ }^{a}$ BMI: Body Mass Index; ${ }^{b}$ Worker: Include part-time job and school attendance; ${ }^{\mathrm{c}}$ Non-worker: Include full-time homemakers and workers on a child-care leave; ${ }^{\mathrm{d}}$ EPDS: Edinburgh Postnatal Depression Scale: a higher score is a tendency for postpartum depression.

Table 2. SF-36v2 subscales, SF-36v2 summary scores, RSES and EPDS for the intervention and control groups.

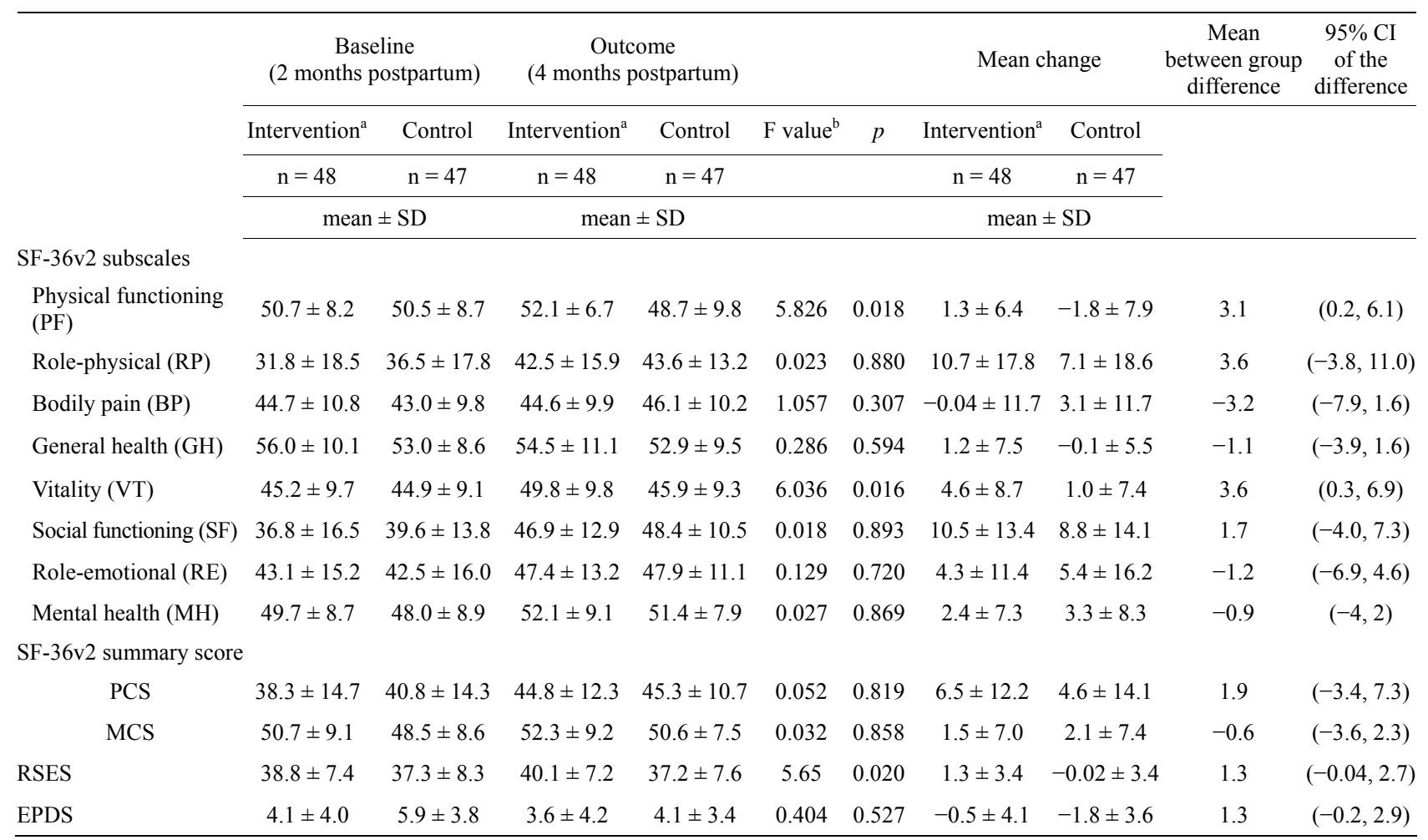

PCS: Physical Component Summary, MCS: Mental Component Summary; RSES: Rosenberge Self-Esteem Scale; EPDS: Edinburgh Postnatal Depression Scale; ${ }^{a}$ Intervention group participated in execise program at 3 months postpartum; ${ }^{\mathrm{b}}$ ANCOVA: analysis of covariance with baseline data as covariate. 
the control group for two of the eight subscales within the SF-36v2: the Physical Functioning (PF) domain ( $\mathrm{p}=$ $0.022)$; and the Vitality (VT) domain $(\mathrm{p}=0.008)$. MCS summary score and other subscales showed no significant differences due to exercise intervention. A significant increase in self-esteem level (RSES) was seen in the 44 women in the exercise intervention group compared to controls $(p=0.037)$. No discrepancy was identified between these results and the ITT analysis of outcome measurements.

\section{DISCUSSION}

This study demonstrated the effect of an exercise program on health-related QOL and self-esteem in women at three months postpartum. PF score and VT score of SF36v2 that is, health-related QOL increased due to exercise in the postpartum period, rather than as a result of natural recovery and this may explain the effects of the intervention. The increase of these subscales might reflect that participating women were doing well after childbirth. We found that postpartum exercise might increase the physiological aspect of health-related QOL. Although the mean of PCS summary score increased 6.5 points after the exercise program in the intervention group, on the other hand, a naturally steeper recovery was found in the control group at the same time. Therefore, the mean between group differences was only 1.3 points.

SF-36v2 standard values for Japanese women in their 30s are as follows: PCS: 51.3 (7.8), 47.6 - 52.7; MCS: 47.9 (10.4), $39.7-56.6$; mean (SD), $25^{\text {th }}-75^{\text {th }}$ percentile. Mean values of PCS for our participants were below the $25^{\text {th }}$ percentile of the coeval general population. Postpartum women seem likely to be experiencing some degree of physical discomfort. For this reason, improving the subscales [physical functioning, role-physical, bodily pain, vitality, general health, social functioning] comprising PCS score among postpartum women is significant. Meanwhile, MCS scores for our participants were at almost the same level as the coeval general population.

The mean of EPDS scores reduced in both intervenetion and control groups, and there were no differences between the two groups due to less change in healthy subjects in our study. The exercise program in this study involved aerobic exercise and it was expected that it would have an effect on reducing postpartum depressive symptoms. However, almost all participants in our study were women without high EPDS scores and significant differences were not found. As another reason for nonsignificant differences, the baseline EPDS score was higher in the control group than in the intervention group, even though we conducted a random allocation. Therefore, control group scores had a potentially steeper de- cline over time compared to the intervention group.

The target population for our study was healthy women during the postpartum period, since we are interested in developing programs for the primary prevention of postpartum depression. Our findings were thus limited to healthy postpartum women. The present intervention did not improve EPDS scores compared to the control group. Speculation on possible effects of programs like this as a treatment for women with depression is beyond the scope of the study.

In future investigation, dose-response trials will need to examine changes in QOL in postpartum women, and the exact type and intensity of exercise that is most appropriate for mothers remain to be determined. A previous RCT that examined the effect of exercise in improveing QOL among sedentary postmenopausal women demonstrated that higher doses of exercise were associated with larger improvements in mental and physical aspects of QOL [14]. Both the intensity and duration of exercise may influence improvements in QOL. As such, it seems desirable to conduct thorough and extensive studies on the effects of physical exercise on postpartum women, although it is difficult for mothers in the child-rearing period to follow an exercise program.

In our study, postpartum exercise resulted in a tendency to increase self-esteem. Self-esteem was the link explaining the relationship between depression and body image, with lower esteem associated with negative body attitude [23]. Many new mothers are confronted with a gap between the actual appearance of their body after childbirth and their expected postpartum weight and body changes. A previous study suggested that mothers' body satisfaction worsened from 1 to 9 months post-partum, and that body dissatisfaction was associated with worse mental health [24]. Another intervention study demonstrated that pregnant adolescents who participated in an aerobic exercise program showed a significant decrease in depressive symptoms over time and an increase in total self-esteem [25]. Thus, it is important to encourage mothers to enhance postpartum self-esteem.

Daley et al. [26] tested the feasibility of an exercise intervention program for women with postpartum depression in a pilot RCT and found comparatively similar results to ours. The intervention group showed a significantly higher self-efficacy for exercise compared to usual care, but the program did not improve depression scores. Assessment of the results suggested that it was difficult to increase exercise participation over the 12 week period due to women's motivation. Especially, in the case of mothers with depressive symptoms, the acceptability of the intervention was important in addition to the effecttiveness of the program. Any intervention designed to include high risk mothers with depression may have a real chance to reduce depressive symptoms. 
The attendance rate in this exercise program was comparatively high and remained at almost $90 \%$ despite many constraints against leaving home in the first six months after childbirth. The program was formulated so that mothers were easily able to attend classes with their babies, and run on a short term basis. The accessibility and acceptability of the services may have contributed to the high attendance rate.

There are several methodological issues that should be considered in interpreting these findings. First, although we tried to enroll a sufficient number of participants, the number was insufficient for power analysis, though the size of our sample was larger than similar previous trials. Second, most participants had normal BMI and were urban dwellers. Therefore, we do not know whether the results could be applied to other populations of postpartum women. Regardless of these limitations, our trial demonstrated that the exercise program used in this study allowed participation at a high rate and was well tolerated by postpartum women, resulting in increased psychological well-being such as health-related QOL and self-esteem.

\section{ACKNOWLEDGEMENTS}

The authors thank all mothers who participated in this study, as well as the staff members of Tokyo University Hospital and Bunkyo-ku Public Health Centers where the study was conducted. We would also like to thank the exercise instructors, Ms. Mako Yoshioka and her colleagues. Our appreciation also goes to Dr. Stuart Gilmour from The University of Tokyo, for advice on the English text. This study was part of a research project funded by a grant from Japan Society for the Promotion of Science (Grant-in-Aid for Scientific Research (B), 20062008, No. 17390577).

\section{REFERENCES}

[1] Gavin, N.I., Gaynes, B.N., Lohr, K.N., Meltzer-Brody, S., Gartlehner, G. and Swinson, T. (2005). Perinatal depression: A systematic review of prevalence and incidence. Obstetrics \& Gynecology, 106, 1071-1083. doi:10.1097/01.AOG.0000183597.31630.db

[2] Okano, S., Sugiyama, T. and Nishiguchi, H. (2007) A screening system for postnatal depression in Japanese primary health care. Japanese Journal of Maternal Health, 48, 16-20. (in Japanese)

http://ci.nii.ac.jp/naid/110006274310

[3] Hoedjes, M., Berks, D., Vogel, I., Franx, A., Visser, W., Duvekot, J.J., Habbema, J.D., Steegers, E.A. and Raat, H. (2011) Symptoms of post-traumatic stress after preeclampsia. Journal of Psychosomatic Obstetrics \& Gynecology, 32, 126-134. doi:10.3109/0167482X.2011.599460

[4] Nagata, M., Ito, K., Suzuki, A. and Matsuura, K. (2007) Application of the EPDS scale for maternal and child health activity in local settings-Follow-up results from neonatal visits to three-month-infant health check-ups.
Japanese Journal of Maternal Health, 48, 289-294. (in Japanese)

[5] Ministry of Health, Labour and Welfare (2007) http://www.mhlw.go.jp/toukei/saikin/hw/k-tyosa/k-tyosa0 7/1-3.html

[6] Deslandes, A., Moraes, H., Ferreira, C., Veiga, H., Silveira, H., Mouta, R., Pompeu, F.A., Coutinho, E.S. and Laks J. (2009) Exercise and mental health: Many reasons to move. Neuropsychobiology, 59, 191-198. doi: $10.1159 / 000223730$

[7] Armstrong, K. and Edwards, H. (2004) The effectiveness of a pram-walking exercise programme in reducing depressive symptomatology for postnatal women. International Journal of Nursing Practice, 10, 177-194. doi:10.1111/j.1440-172X.2004.00478.X

[8] Heh, S.S., Huang, L.H., Ho, S.M., Fu, Y.Y. and Wang, L.L. (2008) Effectiveness of an exercise support program in reducing the severity of postnatal depression in Taiwanese women. Birth, 35, 60-65.

doi:10.1111/j.1523-536X.2007.00192.x

[9] Dritsa, M., Da Costa, D., Dupuis, G., Lowensteyn, I. and Khalifé, S. (2008) Effects of a home-based exercise intervention on fatigue in postpartum depressed women: Results of a randomized controlled trial. Annals of Behavioral Medicine, 35, 179-187. doi:10.1007/s12160-008-9020-4

[10] Da Costa, D., Lowensteyn, I., Abrahamowicz, M., IonescuIttu, R., Dritsa, M., Rippen, N., Cervantes, P. and Khalifé, S. (2009) A randomized clinical trial of exercise to alleviate postpartum depressed mood. Journal of Psychosomatic Obstetrics \& Gynecology, 30, 191-200. doi:10.1080/01674820903212136

[11] Norman, E., Sherburn, M., Osborne, R.H. and Galea, M.P. (2010) An exercise and education program improves well-being of new mothers: A randomized controlled trial. Physical Therapy, 90, 348-355. doi: $10.2522 / \mathrm{ptj} .20090139$

[12] Cramp, A.G. and Bray, S.R. (2009) Postnatal women's feeling state responses to exercise with and without baby. Maternal and Child Health Journal, 14, 343-349. doi:10.1007/s10995-009-0462-5

[13] Montoya Arizabaleta, A.V., Orozco Buitrago, L., Aguilar de Plata, A.C., Mosquera Escudero, M. and RamirezVelez, R. (2010) Aerobic exercise during pregnancy improves health-related quality of life: A randomised trial. Journal of Physiotherapy, 56, 253-258. doi:10.1016/S1836-9553(10)70008-4

[14] Martin, C.K., Church, T.S., Thompson, A.M., Earnest, C.P. and Blair, S.N. (2009) Exercise dose and quality of life: A randomized controlled trial. Archives of Internal Medicine, 169, 269-278. doi:10.1001/archinternmed.2008.545

[15] Devereux, K., Robertson, D. and Briffa, N.K. (2005) Effects of a water-based program on women 65 years and over: A randomised controlled trial. The Australian Journal of Physiotherapy, 51, 102-108. doi:10.1016/S0004-9514(05)70038-6

[16] Fukuhara, S. and Suzukamo, Y. (2004) Manual of SF36v2 Japanese version. Institute for Health Outcomes \& 
Process Evaluation Research, Kyoto.

[17] Haley, S.M., McHorney, C.A. and Ware Jr., J.E. (1994) Evaluation of the MOS SF-36 physical functioning scale (PF-10): I. Unidimensionality and reproducibility of the Rasch item scale. Journal of Clinical Epidemiology, 47, 671-684. doi:10.1016/0895-4356(94)90215-1

[18] Rosenberg, M. (1965) When dissonance fails: On eliminating evaluation apprehension from attitude measurement. Journal of Personality and Social Psychology, 95, 28-42. doi:10.1037/h0021647

[19] Yamamoto, M., Matsui, Y. and Yamanari, Y. (1982) The structure of perceived aspects of self. Japanese Journal of Educational Psychology, 30, 64-68. (in Japanese)

[20] Cox, J.L., Holden, J.M. and Sagovsky, R. (1987) Detection of postnatal depression. Development of the 10-item Edinburgh postnatal depression scale. The British Journal of Psychiatry, 150, 782-786. doi:10.1192/bjp.150.6.782

[21] Okano, T., Murata, M., Masuji, F., Tamaki, R., Nomura, J., Miyaoka, H. and Kitamura, T. (1996) Validation and reliability of a Japanese version of the EPDS. Archives of Psychiatric Diagnosis and Clinical Evaluation, 7, 525533. (in Japanese)

[22] Faul, F., Erdfelder, E., Lang, A.G. and Buchner, A. (2007)
G*Power 3: A flexible statistical power analysis program for the social, behavioral, and biomedical sciences. Behavior Research Methods, 39, 175-191. doi:10.3758/BF03193146

[23] Kamysheva, E., Skouteris, H., Wertheim, E.H., Paxton, S.J. and Milgrom, J. (2008) Examination of a multi-factorial model of body-related experiences during pregnancy: The relationships among physical symptoms, sleep quality, depression, self-esteem, and negative body attitudes. Body Image, 5, 152-163. doi:10.1016/j.bodyim.2007.12.005

[24] Gjerdingen, D., Fontaine, P., Crow, S., McGovern, P., Center, B. and Miner, M. (2009) Predictors of mothers' postpartum body dissatisfaction. Women Health, 49, 491504. doi:10.1080/03630240903423998

[25] Koniak-Griffin, D. (1994) Aerobic exercise, psychological well-being, and physical discomforts during adolescent pregnancy. Research in Nursing \& Health, 17, 253263. doi: $10.1002 /$ nur.4770170404

[26] Daley, A., Winter, H., Grimmett, C., McGuinness, M., McManus, R. and MacArthur, C. (2008) Feasibility of an exercise intervention for women with postnatal depression: A pilot randomised controlled trial. British Journal of General Practice, 58, 178-183. 